\title{
Reading human relationships from their interaction with an interactive humanoid robot
}

\author{
Takayuki Kanda $^{1}$ and Hiroshi Ishiguro ${ }^{1,2}$ \\ ${ }^{1}$ ATR, Intelligent Robotics and Communication Laboratories \\ 2-2-2 Hikaridai Sorakugun Seikacho, 6190288 Kyoto, Japan \\ kanda@atr.co.jp \\ http: //www.irc.atr.co.jp/ \\ ${ }^{2}$ Osaka University, Graduate School of Engineering, Dept. Adaptive Machine Systems \\ Osaka, Japan \\ ishiguro@ams.eng.osaka-u.ac.jp
}

\begin{abstract}
This paper reports our novel approach to developing a social robot. Such a robot reads human relationships from their physical behavior. We have developed an interactive humanoid robot that attracts humans to interact with it and, as a result, induces their group behaviors in front of it. In our approach, the robot recognizes friendly relationships among humans by simultaneously identifying each person in the interacting group. We conducted a two-week experiment in an elementary school, in which Robovie demonstrated proven reasonable performance in identifying friendships among the children. We believe this ability to read human relationships is essential to behaving socially.
\end{abstract}

\section{Introduction}

Recent progress in robotics has brought with it a new research direction known as "interaction-oriented robots." These robots are different to traditional task-oriented robots, such as industrial robots, which perform certain tasks in limited applications. Interaction-oriented robots are designed to communicate with humans and to be able to participate in human society. We are trying to develop such an interaction-oriented robot that will exist as a partner in people's daily lives. As well as providing physical support, these robots will supply communication support such as route-guidance.

Several researchers are endeavoring to realize such interaction-oriented robots. Aibo has become the first interactive robot to prove successful on the commercial market [1], since it behaves as if it were a real animal pet. Breazeal et al. developed the face robot Kismet, and they are exploring sociable aspects of robots produced through its learning ability [2]. Okuno et al. developed a humanoid head that tracks a speaking person with visual and auditory data. In addition, they controlled the personality of the robot by changing the tracking parameter [3]. Burgard et al. developed a museum tour guide robot [4] that was equipped with robust navigational skills and behaved as a museum orientation tool. These research efforts also seem to be devoted to social robots that are embedded in human society. 
Humans have the natural ability to read others' intentions, which is widely known as the joint-attention mechanism in developmental psychology [5], and we believe that this is an essential function for both humans and robots to be social. Scassellati developed a robot with a joint-attention mechanism that follows others' gazes in order to share attention [6]. Kozima et al. also developed a robot with a joint-attention mechanism [7]. In other words, these robots read humans' intentions from their behaviors. Furthermore, a robot system can estimate humans' subjective evaluation of it by observing the humans' body movements [8]. However, these research works mainly focused on the social behaviors among two or three people. Little robotics research work has been attempted to handle social behavior within greater human society. To enable a robot to be social, we believe it is important for the robot to read relationships among humans.

In sociology, sociometric (a matrix that represents relationships) and socio-gram (a direct graph that illustrates the sociometric) methods have been used to represent the relationships that occur among humans. A sociometric test is a subjective test that retrieves the relationships, and lets a human directly answer the name of others whom he/she likes and dislikes. It has been widely used to determine the relationships in a classroom or a company; however, it has recently become difficult to apply (in particular, asking the name of disliked persons), since it might promote negative relationships.

In the field of computer science, several research works have analyzed human relationships. Eveland et al. analyzed online communication on a CSCW (computersupported collaborative work) system [9]. They plotted each user's data on a sociogram according to the amount of online communication among them. Nomura and her colleagues developed a Web-analyzing system to retrieve humans' online relationships from hyperlinks in their web pages [10]. Watts and Strogatz conducted a computer simulation to find a simple model for global human society and proposed that small-world networks represent large-scale human relationships [11].

In this paper, we report our approach to reading human relationships with an interactive robot, an ability that is probably essential for interactive robots to be social. We have developed an interactive humanoid robot, named Robovie, that autonomously interacts with humans. As a result, the robot attracts humans to interact with it and induces humans' group behaviors in front of it. In our approach, the robot recognizes friendly relationships among humans by simultaneously identifying each person in the interacting group. We conducted a two-week experiment in an elementary school, in which Robovie demonstrated reasonable performance in identifying friendships among the children.

\section{Robovie: An Interactive Humanoid Robot}

\subsection{Hardware}

Figure 1 shows the humanoid robot "Robovie" [12]. The robot is capable of human-like expression and recognizes individuals by using various actuators and sensors. Its body 


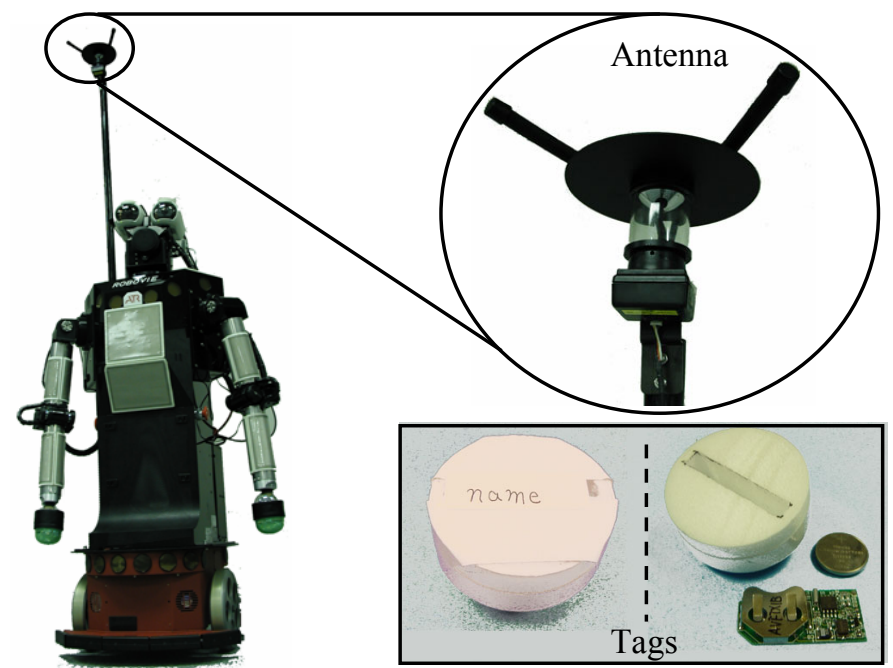

Figure 1: Robovie (left) and Wireless tag

Robovie is an interactive humanoid robot that autonomously speaks, makes gestures, and moves around. With its antenna and tags, it is able to identify individuals.

possesses highly articulated arms, eyes, and a head, which were designed to produce sufficient gestures to communicate effectively with humans. The sensory equipment includes auditory, tactile, ultrasonic, and vision sensors, which allow the robot to behave autonomously and to interact with humans. All processing and control systems, such as the computer and motor control hardware, are located inside the robot's body.

\subsection{Person identification with wireless ID tags}

To identify individuals, we used a wireless tag system capable of multi-person identification by partner robots (Detailed specification and system configuration is described in [13]). Recent RFID (radio frequency identification) technologies have enabled us to use contact-less identification cards in practical situations. In this study, children were given easy-to-wear nameplates $(5 \mathrm{~cm}$ in diameter) in which a wireless tag was embedded. A tag (Fig. 1, lower-right) periodically transmitted its ID to the reader installed on the robot. In turn, the reader relayed received IDs to the robot's software system. It was possible to adjust the reception range of the receiver's tag in real-time by software. The wireless tag system provided the robots with a robust means of identifying many children simultaneously. Consequently, the robots could show some human-like adaptation by recalling the interaction history of a given person.

\subsection{Interactive behaviors}

"Robovie" features a software mechanism for performing consistent interactive behaviors (detailed mechanism is described in [14]). The objective behind the design of Robovie is that it should communicate at a young child's level. One hundred interactive behaviors have been developed. Seventy of them are interactive behaviors such 
as shaking hands, hugging, playing paper-scissors-rock, exercising, greeting, kissing, singing, briefly conversing, and pointing to an object in the surroundings. Twenty are idle behaviors such as scratching the head or folding the arms, and the remaining 10 are moving-around behaviors. In total, the robot could utter more than 300 sentences and recognize about 50 words.

Several interactive behaviors depended on the person identification function. For example, there was an interactive behavior in which the robot called a child's name if that child was at a certain distance. This behavior was useful for encouraging the child to come and interact with the robot. Another interactive behavior was a body-part game, where the robot asked a child to touch a body part by saying the part's name.

The interactive behaviors appeared in the following manner based on some simple rules. The robot sometimes triggered the interaction with a child by saying "Let's play, touch me," and it exhibited idling or moving-around behaviors until the child responded; once the child reacted, it continued performing friendly behaviors for as long as the child responded. When the child stopped reacting, the robot stopped the friendly behaviors, said "good bye," and re-started its idling or moving-around behaviors.

\section{Reading Humans' Friendly Relationships}

\subsection{Basic ideas}

Our approach consists of the two functions described below (Figure 2). Since humans have friendly relationships, they behave in a group. Meanwhile, a robot induces spontaneous group behavior with its interactive behaviors.

\subsubsection{Group behavior and friendship}

Like and dislike are two of the essential relationships among humans. Humans change their opinions based on like and dislike relationships, which is well-known as Heider's balance theory [15]. For example, if a person's friend has an opposing opinion, the person would change his/her opinion to be agreeable with the friend's opinion. Humans establish friendship based on their mutual "like" relationships of each other. In developmental psychology, Ladd et al. found that even children form their own group and behave with the group based on their friendship [16]. In other words, if we observe such a group's behavior, we can estimate the friendships among the members.

\subsubsection{Use of interactive robot to cause spontaneous group behavior}

Our interactive humanoid robot Robovie autonomously interacts with humans. By executing interactive behaviors, the robot attracts humans to interact with it; on the other hand, humans often behave in a group, so the robot induces human group behaviors in front of it. As a result, the robot can recognize friendly relationships among humans by simultaneously identifying each person in the interacting group. 


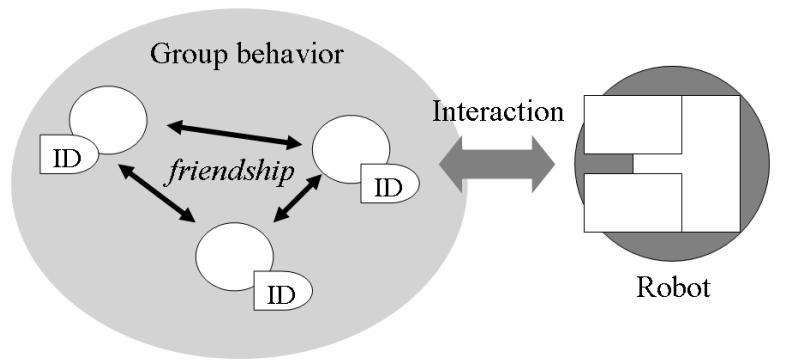

Figure 2: Mechanism of reading humans' friendly relationships

Robot identifies multiple people in front of it simultaneously; as a result, it recognizes friendship among them, because the robot's interactive behaviors cause the group behavior.

We might read such friendly relationships by simply observing humans' group behavior in their daily life. However, humans sometimes behave as a group because it is necessary or required. For example, the activity "humans collaborate to carry a heavy box" does not always indicate friendly relationships among them. Thus, we believe that it is better to read human relationships by observing spontaneous group behavior such as interaction with the robot. We believe that in the future robots will carry out various communication tasks in our daily lives such as foreign language education [17], and humans will freely interact with robots even in these applications.

\subsection{Algorithm}

From a sensor (in this case, wireless ID tags and receiver), the robot constantly obtains the IDs (identifiers) of individuals who are in front of it. The robot continuously accumulates its interacting time with person $A\left(T_{A}\right)$ and the time that person $A$ and $B$ simultaneously interact with it $\left(T_{A B}\right.$, which is equivalent to $\left.T_{B A}\right)$. We define the estimated friendship from person $A$ to $B(\operatorname{Friend}(A \rightarrow B))$ as

$$
\begin{gathered}
\text { Friend }(A \rightarrow B)=i f\left(T_{A B} / T_{A}>T_{T H}\right), \\
T_{A}=\sum i f\left(\text { observe }(A) \text { and }\left(S_{t}<S_{T H}\right)\right) \cdot \Delta \mathrm{t}, \\
T_{A B}=\sum i f\left(\text { observe }(A) \text { and } \operatorname{observe}(B) \text { and }\left(S_{t}<S_{T H}\right)\right) \cdot \Delta \mathrm{t},
\end{gathered}
$$

where observe( $A$ ) becomes true only when the robot observes the ID of person $A$, if() becomes 1 when the logical equation inside the bracket is true (otherwise 0 ), and $T_{T H}$ is a threshold of simultaneous interaction time. We also prepared a threshold $S_{T H}$, and the robot only accumulates $T_{A}$ and $T_{A B}$ so that the number of persons simultaneously interacting at time $t\left(S_{t}\right)$ is less than $S_{T H}$ (Eqs. 2 and 3). In our trial, we set $\Delta t$ to one second.

\section{Experiment}

We conducted a field experiment in an elementary school for two weeks with the developed interactive humanoid robot, which was originally designed to promote 
children's English learning. As we reported in [17], the robots had a positive affect on the children. In this paper, we use the interaction data during that trial as a test-set of our approach to reading friendship from the children's interaction.

\subsection{Method}

We performed an experiment at an elementary school in Japan for two weeks. Subjects were sixth-grade students from three different classes, totaling 109 students (1112 years old, 53 male and 56 female). There were nine school days included in those two weeks. Two identical robots were placed in a corridor that connects the three classrooms (Figure 3). Children could freely interact with both robots during recesses (in total, about an hour per day), and each child had a nameplate with an embedded wireless tag so that each robot could identify the child during interaction.

We administered a questionnaire that asked the children to write down the names of their friends. This obtained friendship information was collected for comparison with the friendship relationships estimated by our proposed method.

\subsection{Results}

\subsubsection{Evaluation of Estimated Relationships with Reliability and Coverage}

Since the number of friendships among children was fairly small, we focused on the appropriateness (coverage and reliability) of the estimated relationships. This is similar to the evaluation of an information retrieval technique such as a Web search. Questionnaire responses indicated 1,092 friendships among a total of 11,772 relationships; thus, if we suppose that the classifier always classifies a relationship as a nonfriendship, it would obtain $90.7 \%$ correct answers, which means the evaluation is completely useless. Thus, we evaluate our estimation of friendship based on reliability and coverage, which are defined as follows.
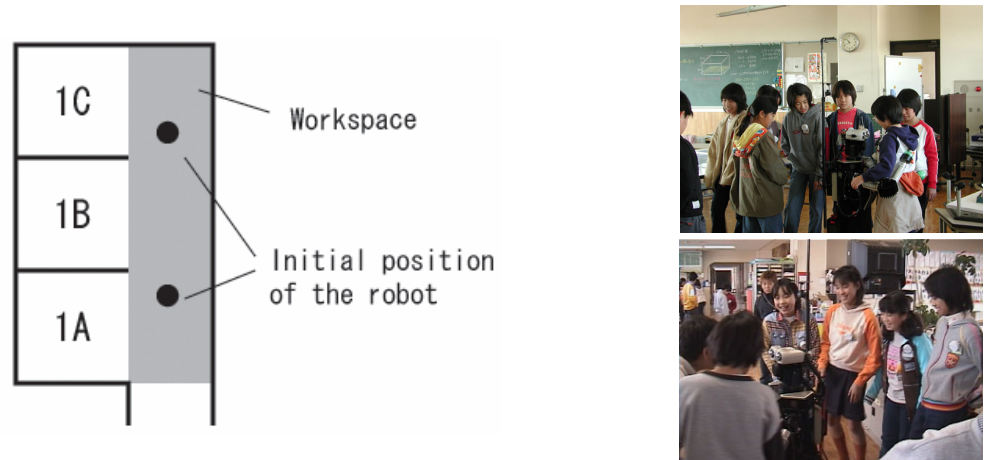

Figure 3: Environment of the elementary school where we installed the robot.

Left figure is a map of the environment, and right photos are scenes of the experiment. 
Table 1: Estimation results with various parameters

\begin{tabular}{|c|c|c|c|c|c|c|c|}
\hline \multirow{2}{*}{\multicolumn{2}{|c|}{$\begin{array}{l}\text { coverage } \\
\text { reliability }\end{array}$}} & \multicolumn{6}{|c|}{$T_{T H}$ (simultaneously interacting time) } \\
\hline & & 0.3 & 0.2 & 0.1 & 0.05 & 0.01 & 0.001 \\
\hline \multirow{4}{*}{ 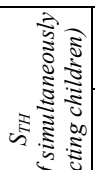 } & 2 & 0.01 & 0.02 & 0.03 & 0.04 & 0.04 & 0.04 \\
\hline & & 1.00 & 0.93 & 0.79 & 0.59 & 0.54 & 0.54 \\
\hline & 5 & 0.00 & 0.02 & 0.06 & 0.11 & 0.18 & 0.18 \\
\hline & & 1.00 & 1.00 & 0.74 & 0.47 & 0.29 & 0.28 \\
\hline \multirow{2}{*}{ 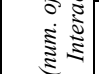 } & 10 & 0.00 & 0.00 & 0.04 & 0.13 & 0.29 & 0.31 \\
\hline & & & 1.00 & 0.74 & 0.46 & 0.23 & 0.20 \\
\hline
\end{tabular}

('-' indicates that no relationships were estimated, so reliability was not calculated)

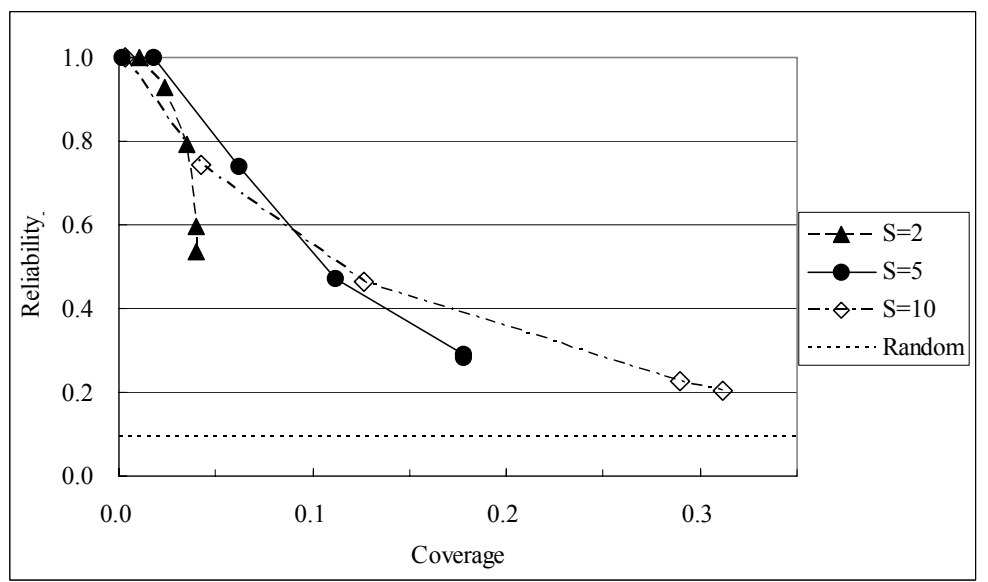

Figure 4: Illustrated estimation results with various parameters

(Each line corresponds with the $S_{T H}(2,5$, and 10). Each point of these lines corresponds with a certain $T_{T H}$ in Table 1.)

Reliability $=$ number of correct friendships in estimated friendships / number of estimated friendships

Coverage $=$ number of correct friendships in estimated friendship / number of friendships from the questionnaire

Table 1 and Fig. 4 indicate the results of estimation with various parameters $\left(S_{T H}\right.$ and $T_{T H}$ ). In Fig. 4, random represents the reliability of random estimation where we assume that all relationships are friendships (since there are 1,092 correct friendships among 11,772 relationships, the estimation obtains $9.3 \%$ reliability with any coverage). In other words, random indicates the lower boundary of estimation. Each of the other lines in the figure represents the estimation result with different $S_{T H}$, which has several points corresponding to different $T_{T H}$. There is obviously a tradeoff between reliability and coverage, which is controlled by $T_{T H} ; S_{T H}$ has a small effect on the tradeoff, $S=5$ mostly performs better estimation of the friendship, and $S=10$ performs better estimation when coverage is more than 0.15 . As a result, our method successfully estimated $5 \%$ of the friendship relationships with greater than $80 \%$ accuracy (at " $\mathrm{S}=5$ ") and $15 \%$ of them with nearly $50 \%$ accuracy (at " $\mathrm{S}=10$ "). 
Table 2: Gender effect (at $\mathrm{S}=5$ )

\begin{tabular}{|l|r|r|r|r|r|r|}
\hline \multirow{2}{*}{ reliability } & \multicolumn{6}{|c|}{$T_{T H}$} \\
\cline { 2 - 7 } & 0.3 & 0.2 & 0.1 & 0.05 & 0.01 & 0.001 \\
\hline Male-Male & - & 1.00 & 0.81 & 0.60 & 0.45 & 0.44 \\
Female-Female & 1.00 & 1.00 & 0.80 & 0.61 & 0.43 & 0.41 \\
Male-Female & - & - & 0.20 & 0.03 & 0.01 & 0.01 \\
\hline
\end{tabular}

('-' indicates that no relationships were estimated, so reliability was not calculated)

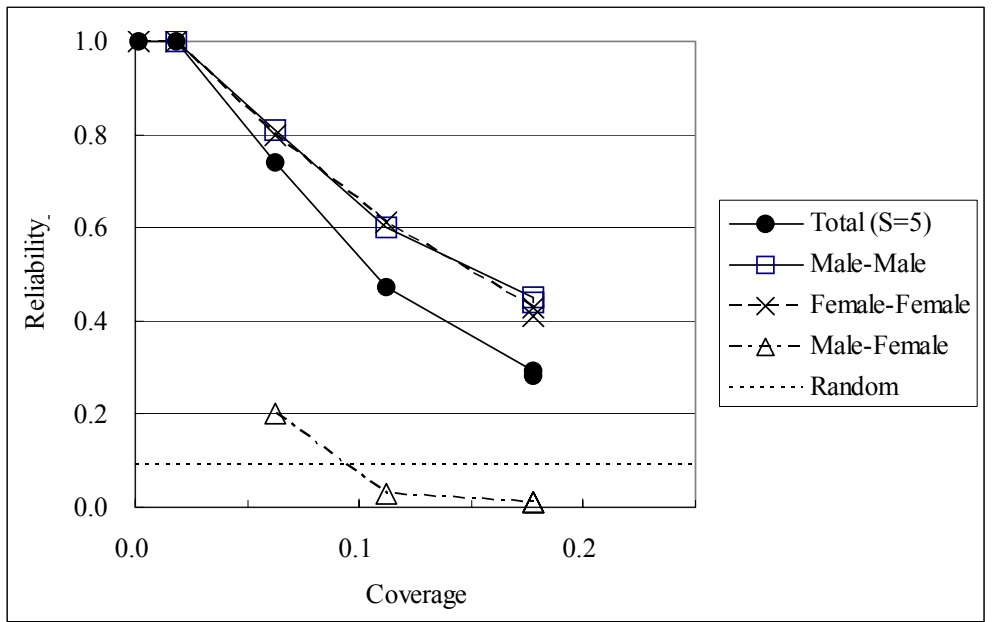

Figure 5: Illustration of gender effects at $\mathrm{S}_{\mathrm{TH}}=5$

(Each point of these lines corresponds with a certain $T_{T H}$ in Table 2. The plotted coverage of all groups is based on the coverage of "total" group, which is equal to " $S_{T H}=5$ " in Table 1.)

\subsubsection{Gender effects}

To verify the appropriateness of the estimation in detail, we analyzed gender effects. We first classified the relationships into three groups: male-male, female-female, and male-female (including both male to female and female to male). Then we calculated the reliability and coverage for the relationships of the three groups. Table 2 and Fig. 5 show the result at $\mathrm{S}=5$. The male-male and female-female groups indicate better performance than the total case, while the male-female group indicated extremely low performance; in fact, the performance of the male-female group was lower than random performance, because the children rarely reported friendships with the opposite gender. We believe they might hesitate to report friendly relationships with the opposite gender, which would cause the lower performance of our estimation. Meanwhile, this also suggests the positive prospects of our approach because it might be able to estimate friendly relationships that a subjective questionnaire cannot detect.

\section{Discussion and Conclusions}

We have developed an interactive robot that reads humans' friendly relationships. This was accomplished by the robot inducing friendly group behaviors in front of it 
and simultaneously identifying multiple people. Experimental results show that our system successfully estimated $5 \%$ of the friendly relationships (retrieved by subjective questionnaire) with greater than $80 \%$ accuracy, and $15 \%$ of them with nearly $50 \%$ accuracy. We believe that this is a reasonable performance, although the estimation algorithm is very simple. In addition, the results suggest that the children hesitated to answer whether they had friendly relationships with individuals of the opposite gender, which agrees with our estimation. In other words, in some cases observing the children's interaction probably leads to better acquisition of knowledge about their friendship than a traditional subjective method. We believe that these results demonstrate the promising potential of our approach. This ability of reading human relationships will be essential and useful for social robots. For example, if robots can guess human friendship relationships, they will be able to promote the relationships, join the relationships or reconcile bullying problems.

There was a tradeoff between reliability and coverage of the estimated relationships. The strict threshold for the time when people simultaneously interact resulted in a small amount of estimation with high accuracy, whereas a moderated threshold provided a larger amount of estimation with low accuracy. However, even if we had moderated the threshold, we still could not estimate more than $30 \%$ of the friendship relationships. We do not consider this as an upper limitation of estimation. Instead, we believe the amount of data (that is, the interaction between the children and the robot) was not sufficient for all friendships to be observed by the robot. To verify this hypothesis, an important future work is to gather more long-term interaction data as well as to improve the estimation accuracy by finding other effective rules.

Another concern might be the applicability of this approach. In this paper, we applied our interactive robot that behaves like a child to elementary school students. It is not yet clear whether this approach can be extended to general society that includes adults; we do believe, however, that if robots have the ability to keep interacting with adults, they will even be able to estimate adults' friendly relationships, because social behavior during spontaneous interactions seems not to differ between children and adults. For example, they behave in a group based on their friendships. Thus, if we can develop an interactive robot that can interact with adults for a reasonable period of time, such as by giving interesting information and chattering, we can extend our approach to more general society that includes adults.

\section{Acknowledgements}

We wish to thank the teachers and students at the elementary school for their agreeable participation and helpful suggestions. We also thank Takayuki Hirano and Daniel Eaton, who helped with this field trial in the elementary school. This research was supported in part by the Telecommunications Advancement Organization of Japan. 


\section{References}

1. Fujita, M.: AIBO; towards the era of digital creatures, Int. J. of Robotics Research, Vol. 20, No. 10 (2001) 781-794.

2. Breazeal, C. and Scassellati, B.: A context-dependent attention system for a social robot, Proc. Int. Joint Conf. on Artificial Intelligence (1999) 1146-1151.

3. Okuno, H. G., Nakadai, K., and Kitano, H.: Realizing audio-visually triggered ELIZA-like non-verbal behaviors, PRICAI2002, LNAI 2417, Lecture Notes in Artificial Intelligence, Springer-Verlag (2002) 552-562.

4. Burgard, W., Cremers, A. B., Fox, D., Hahnel, D., Lakemeyer, G., Schulz, D., Steiner, W., and Thrun, S.: The interactive museum tour-guide robot, Proc. of National Conference on Artificial Intelligence (1998) 11-18.

5. Moore, C. and Dunham, P. J. eds.: Joint Attention: Its Origins and Role in Development, Lawrence Erlbaum Associates (1995).

6. Scassellati, B.: Investigating Models of Social Development Using a Humanoid Robot, Biorobotics, MIT Press (2000).

7. Kozima, H. and Vatikiotis-Bateson, E.: Communicative criteria for processing time/spacevarying information, Proc. IEEE Int. Workshop on Robot and Human Communication (2001).

8. Kanda, T., Ishiguro, H., Imai, M., and Ono, T.: Body Movement Analysis of Human-Robot Interaction, International Joint Conference on Artificial Intelligence (2003) 177-182.

9. Eveland, J. D. and Bikson T. K.: Evolving electronic communication networks: an empirical assessment, Proceedings of the 1986 ACM conference on Computer-supported cooperative work (1986) 91-101.

10. Nomura S., Oyama S., Hayamizu T., and Ishida T.: Analysis and Improvement of HITS Algorithm for Detecting Web Communities, The 2002 International Symposium on Applications and the Internet (2002) 132-140.

11. Watts, D. J. and Strogatz, S. H.: Collective dynamics of 'small-world' networks, Nature, Volume 393, Issue 6684 (1998) 440-442.

12. Ishiguro, H., Ono, T., Imai, M., and Kanda, T.: Development of an interactive humanoid robot "Robovie" -An interdisciplinary approach, R. A. Jarvis and A. Zelinsky (eds.), Robotics Research, Springer (2003) 179-191.

13. Kanda, T., Hirano, T., Eaton, D., and Ishiguro, H.: Person Identification and Interaction of Social Robots by Using Wireless Tags, IEEE/RSJ International Conference on Intelligent Robots and Systems (2003).

14. Kanda, T., Ishiguro, H., Imai, M., Ono T., and Mase, K.: A constructive approach for developing interactive humanoid robots, IEEE/RSJ International Conference on Intelligent Robots and Systems (2002) 1265-1270.

15. Heider, F.: The Psychology of interpersonal relations. Wiley (1958).

16. Ladd, G. W., Price, J. M., and Hart, C. H.: Preschooler's behavioral orientations and patterns of peer contact: predictive of peer status?, in Asher S. R. and Coie J. D. (eds.) Peer rejection in childhood, Cambridge University Press (1990) 90-115.

17. Kanda, T., Hirano, T., Eaton, D., and Ishiguro, H.: A practical experiment with interactive humanoid robots in a human society, Third IEEE International Conference on Humanoid Robots (2003). 